\title{
The Stability of Dust-Laden Vortices
}

\author{
A. D. Railton ${ }^{1, a}$ and J.C. B. Papaloizou ${ }^{1}$ \\ ${ }^{1}$ University of Cambridge
}

\begin{abstract}
We formulate a procedure for finding equilibrium solutions for vortices in the shearing sheet with arbitrary vorticity and density profiles and then testing their stability to linear perturbations.
\end{abstract}

\section{Introduction}

The idea that planets could form in anticyclonic vortices in proto-planetary (PP) discs was first suggested by Barge and Sommeria in 1995 [1]. Since then it was found that a field of vortices could indeed produce inhomogeneous particle distributions [2] and that anticyclonic vortices could form coherent and long-lived structures $[3,4]$. There are also a plethora of instabilities capable of producing these vortices in discs. Here we investigate whether the presence of dust in a vortex stabilises it or not. This problem was first looked at by Chang and Oishi [5] who claim to have found a 'heavy-core' instability, a 2D, linear, parametric instability which appears in elliptic PP disc vortices with steep enough density gradients and large enough aspect ratios. However, this instability has never been seen in any numerical simulations of dust-loaded vortices [e.g. 6, 7]. Also, noting that [5] did not employ a vortex that was consistently in equilibrium, we report preliminary studies that revisit this issue.

\section{The Equilibrium Model}

\subsection{The Governing Equations}

We begin by modelling out disc as a $2 \mathrm{D}$ structure obeying the inviscid Navier-Stokes equations in a frame rotating with constant angular velocity $\Omega=\Omega \hat{\mathbf{z}}$. We define a streamfunction $\psi$ such that our velocity field $\mathbf{u}$ satisfies $\mathbf{u}=\nabla \times \psi \hat{\mathbf{z}}$. Writing the gravitational and centrifugal terms as a combined potential $\tilde{\Phi}=-\frac{3}{2} \Omega^{2} r^{2}$ the time-independent Navier-Stokes equations can be written thus:

$$
\nabla\left(\frac{P}{\rho}+\tilde{\Phi}+\frac{1}{2}|\nabla \psi|^{2}\right)=-\left(\omega+2 \Omega+\frac{P}{\rho^{2}} \frac{d \rho}{d \psi}\right) \nabla \psi \quad \Rightarrow \quad \nabla F=-Q \nabla \psi \quad \Rightarrow \quad \frac{d F}{d \psi}=-Q
$$

where $\omega=-\nabla^{2} \psi$ is the flow's vorticity. We move to the shearing sheet approximation detailed in [8], such that $x=r-r_{0}$ and $y=\phi$ define the local coordinate system. We write the streamfunction $\psi$

\footnotetext{
ae-mail: ar488@cam.ac.uk
} 
as sum of the background, Keplerian flow and a perturbation so $\psi=\psi_{0}+\psi_{1}$. With the background obeying $\psi_{0}=\frac{3}{4} \Omega x^{2}$ a little algebraic manipulation and we arrive at

$$
\nabla^{2} \psi_{1}=\frac{d F_{1}}{d \psi}+\frac{P}{\rho^{2}} \frac{d \rho}{d \psi}=\mathcal{A}(\psi)+\frac{P}{\rho} \mathcal{B}(\psi)
$$

We call $\mathcal{A}(\psi)$ our vorticity source and $\mathcal{B}(\psi)$ our density source. We find the pressure distribution $\frac{P}{\rho}$ by integrating equation (1) with respect to $\psi$, so

$$
\frac{P}{\rho}=\frac{3}{2} \Omega^{2} x^{2}-\frac{1}{2}|\nabla \psi|^{2}-2 \Omega \psi+\int\left[\nabla^{2} \psi-\frac{P}{\rho^{2}} \frac{d \rho}{d \psi}\right] d \psi .
$$

In this one-fluid model our dust is assumed to be completely coupled to the gas and appears as the background density $\rho_{0}=\rho_{0}(\psi)$ which may be specified as an arbitrary function.

\subsection{Numerical Treatment}

Our approach when integrating the Poisson equation (2) numerically is to prescribe both the vorticity source $\mathcal{A}$ and the density source $\mathcal{B}$ to be non-vanishing strictly only within our vortex. We then start with an initial guess of where the bounding streamline will be and solve equation (2) iteratively using the 2D Green's Function:

$$
\psi(\mathbf{r})=\frac{1}{2 \pi} \iint \log \left|\mathbf{r}-\mathbf{r}^{\prime}\right|\left[\mathcal{A}(\psi)+\frac{P}{\rho} \mathcal{B}(\psi)\right] d^{2} \mathbf{r}^{\prime} .
$$

$P / \rho$ must be calculated at each numerical integration step from $\psi$ using equation (3) then included in the next iteration of the integration of (2) until we converge on the equilibrium solution we require.

\section{Stability Analysis}

\subsection{The Perturbation Equations Governing Horizontal Stability}

With a range of equilibrium solutions calculated we now propose to investigate their linear stability. In the horizontal stability limit in which the vertical wave number $k_{z} \rightarrow \infty$, we can neglect the pressure perturbation $p^{\prime}$ (see [9]) and our perturbed equations become

$$
\begin{aligned}
\frac{D u_{x}^{\prime}}{D t} & =-u_{x}^{\prime} \frac{\partial u_{x 0}}{\partial x}-u_{y}^{\prime} \frac{\partial u_{x 0}}{\partial y}+2 \Omega u_{y}^{\prime}+\frac{\rho^{\prime}}{\rho_{0}^{2}} \frac{\partial P_{0}}{\partial x} \\
\frac{D u_{y}^{\prime}}{D t} & =-u_{x}^{\prime} \frac{\partial u_{y 0}}{\partial x}-u_{y}^{\prime} \frac{\partial u_{y 0}}{\partial y}-2 \Omega u_{x}^{\prime}+\frac{\rho^{\prime}}{\rho_{0}^{2}} \frac{\partial P_{0}}{\partial y} \\
\frac{D \rho^{\prime}}{D t} & =-u_{x}^{\prime} \frac{\partial \rho_{0}}{\partial x}-u_{y}^{\prime} \frac{\partial \rho_{0}}{\partial y}
\end{aligned}
$$

We can write this as a matrix equation by letting $\mathbf{x}=\left(u_{x}^{\prime}, u_{y}^{\prime}, \rho^{\prime}\right)^{\mathrm{T}}$ so $\frac{D \mathbf{x}}{D t}=\mathrm{Mx}$. Matrix $\mathrm{M}$ can be calculated from the numerical equilibrium solutions on each streamline. Changing variables to that of arclength $\sigma$ round a streamline, such that $\left|\mathbf{u}_{0}\right|=\frac{D \sigma}{D t}$ we have the working form of the perturbation equations (5)

$$
\frac{D \mathbf{x}}{D \sigma}=\frac{1}{\left|\mathbf{u}_{0}(\sigma, \psi)\right|} \mathrm{M}(\sigma, \psi) \mathbf{x} .
$$

For each streamline our matrix $\mathrm{M}$ is periodic in arclength $\sigma$ so we can use Floquet analysis to determine the stability of each vortex (see [9] for details). This formalism can be extended to general wavenumbers but details are omitted here for the sake of brevity. 


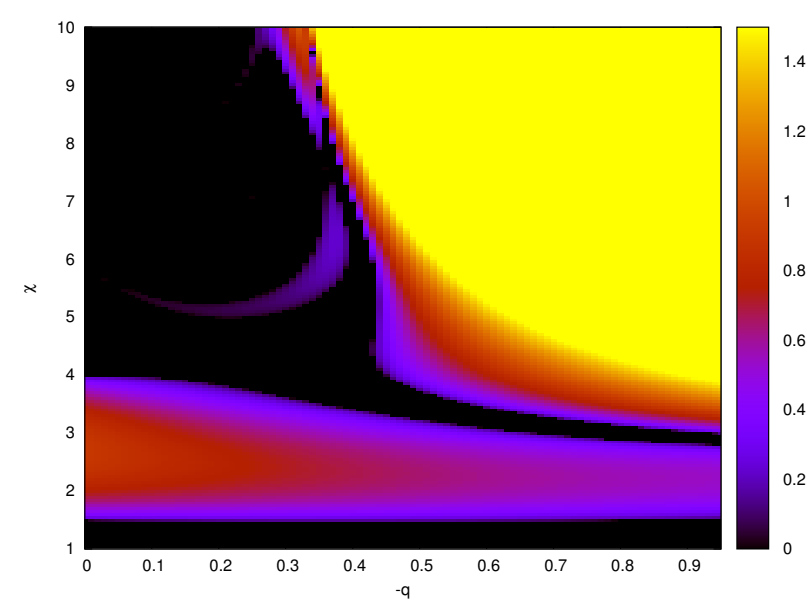

Figure 1. Growth rates for Kida vortices in the $q-\chi$ plane for the horizontal stability $k_{z} \rightarrow \infty$ case. $\chi$ is the aspect ratio of the vortex, and $q$ is related to its density perturbation.

\subsection{The Stability of Kida Vortices}

Kida vortices [10] are the simplest vortex solutions that can be found in the shearing sheet with a simple step function prescription for the vorticity and a flat density profile and elliptic streamlines. For a first calculation we prescribe a background density inside the Kida vortices, neglecting its effect on the streamlines. We so by defining a constant $q \propto \frac{d \log \rho_{0}}{d \psi}$. Since the Kida streamfunction $\psi \propto \chi^{2} x^{2}+y^{2}$, for $q<0$ this gives us a Gaussian density profile $\rho_{0}=A \exp \left[\frac{3 q}{4 S \chi b^{2}}\left(\chi^{2} x^{2}+y^{2}\right)\right]$, where $S$ is the shear rate in the disc (so will be numerically defined) $\chi$ is the aspect ratio of the vortex and $b$ is the semi-major axis of the vortex (set to be 1 in dimensionless units).

In the case of horizontal stability, Figure 1 shows a stable region (albeit punctuated by resonant bands) for small $(-q \lesssim 0.3)$ density perturbations and an aspect ratio $\chi \gtrsim 4$. Future work will extend the analysis to more general cases.

\section{References}

[1] P. Barge, J. Sommeria, Astronomy and Astrophysics 295, L1 (1995)

[2] O. Cardoso, B. Gluckmann, O. Parcollet, P. Tabeling, Physics of Fluids 8, 209 (1996)

[3] A. Bracco, P.H. Chavanis, A. Provenzale, E.A. Spiegel, Physics of Fluids 11, 2280 (1999)

[4] P.H. Chavanis, Astronomy and Astrophysics 356, 1089 (2000)

[5] P. Chang, J.S. Oishi, The Astrophysical Journal 721, 1593 (2010)

[6] S. Inaba, P. Barge, The Astrophysical Journal 649, 415 (2006)

[7] H. Meheut, Z. Meliani, P. Varniere, W. Benz, Astronomy and Astrophysics 545, 134 (2012)

[8] P. Goldreich, D. Lynden-Bell, Monthly Notices of the Royal Astronomical Society 130, 125 (1965)

[9] G. Lesur, J.C.B. Papaloizou, Astronomy and Astrophysics 498, 1 (2009)

[10] S. Kida, Journal of the Physical Society of Japan 50, 3517 (1981) 